Article

Subscriber access provided by King Abdullah University of Science and Technology Library

\title{
Impact of Molecular Orientation and Spontaneous Interfacial Mixing on the Performance of Organic Solar Cells
}

Guy O. Ngongang Ndjawa, Kenneth R. Graham, Ruipeng Li, Sarah M Conron, Patrick Erwin, Kang Wei Chou, George Burkhard, Kui Zhao, Eric T. Hoke, Mark E Thompson, Michael D. McGehee, and Aram Amassian

Chem. Mater., Just Accepted Manuscript • DOI: 10.1021/acs.chemmater.5b01845 • Publication Date (Web): 28 Jul 2015

Downloaded from http://pubs.acs.org on August 2, 2015

\section{Just Accepted}

"Just Accepted" manuscripts have been peer-reviewed and accepted for publication. They are posted online prior to technical editing, formatting for publication and author proofing. The American Chemical Society provides "Just Accepted" as a free service to the research community to expedite the dissemination of scientific material as soon as possible after acceptance. "Just Accepted" manuscripts appear in full in PDF format accompanied by an HTML abstract. "Just Accepted" manuscripts have been fully peer reviewed, but should not be considered the official version of record. They are accessible to all readers and citable by the Digital Object Identifier (DOI®). "Just Accepted" is an optional service offered to authors. Therefore, the "Just Accepted" Web site may not include all articles that will be published in the journal. After a manuscript is technically edited and formatted, it will be removed from the "Just Accepted" Web site and published as an ASAP article. Note that technical editing may introduce minor changes to the manuscript text and/or graphics which could affect content, and all legal disclaimers and ethical guidelines that apply to the journal pertain. ACS cannot be held responsible for errors or consequences arising from the use of information contained in these "Just Accepted" manuscripts. 


\section{Impact of Molecular Orientation and Spontaneous Interfacial Mixing on the Performance of Organic Solar Cells}

Guy O. Ngongang Ndjawa ${ }^{1}$, Kenneth R. Graham ${ }^{1,2}$, Ruipeng Lit ${ }^{1,3}$, Sarah M. Conron ${ }^{4}$, Patrick Erwin', Kang Wei Chou ${ }^{1}$, George Burkhard ${ }^{2}$, Kui Zhao ${ }^{1}$, Eric T. Hoke ${ }^{2}$, Mark E. Thompson ${ }^{4}$, Michael D. McGehee ${ }^{2}$, Aram Amassian ${ }^{1} *$

${ }^{1}$ Physical Sciences and Engineering Division, Solar and Photovoltaic Engineering Research Center, King Abdullah University of Science $\square$ and Technology (KAUST), Thuwal, Saudi Arabia, 23955-6900,*E-mail: aram.amassian@kaust.edu.sa

$20{ }^{2}$ Department of Materials Science and Engineering, Stanford University, Stanford, 21 California 94305, United States,

$22{ }^{3}$ Cornell High Energy Synchrotron Source (CHESS), Cornell University, Ithaca, NY, 23 14850, United States,

$24{ }^{4}$ Department of Chemistry, University of Southern California, Los Angeles, 25 California 90089, United States. 
2 Abstract

3 A critically important question that must be answered to understand how organic solar

4 cells operate and should be improved is how the orientation of the donor and acceptor

5 molecules at the interface influences exciton diffusion, exciton dissociation by

6 electron transfer and recombination. It is exceedingly difficult to probe the orientation

7 in bulk heterojunctions because there are many interfaces and they are arranged with

8 varying angles with respect to the substrate. One of the best ways to study the

9 interface is to make bilayer solar cells with just one donor-acceptor interface. Zinc

10 phthalocyanine is particularly interesting to study because its orientation can be

11 adjusted by using a 2-nm-thick copper iodide seed layer before it is deposited.

12 Previous studies have claimed that solar cells in which fullerene acceptor molecules

13 touch the face of zinc phthalocyanine have more current than ones in which the

14 fullerenes touch the edge of zinc phthalocyanine because of suppressed

15 recombination. We have more thoroughly characterized the system using in situ x-ray

16 photoelectron spectroscopy and x-ray scattering and found that the interfaces are not

17 as sharp as previous studies claimed when formed at room temperature or above.

18 Fullerenes have a much stronger tendency to mix into the face-on films than into the

19 edge-on films. Moreover we show that almost all of the increase in the current with

20 face-on films can be attributed to improved exciton diffusion and to the formation of a

21 spontaneously mixed interface, not suppressed recombination. This work highlights

22 the importance of spontaneous interfacial molecular mixing in organic solar cells, the

23 extent of which depends on molecular orientation of frontier molecules in donor

24 domains. 


\section{Introduction}

4 Organic photovoltaics (OPVs) are emerging as a promising photovoltaic 5 technology because of their potential low cost and ability to be manufactured in a 6 continuous roll-to-roll manner for large-area solar energy harvesting. The power 7 conversion efficiency (PCE) of OPVs has consistently increased over the past 10-15 8 years, with recent PCEs of 10\% for single-junction solar cells and $12 \%$ for multi9 junction solar cells. ${ }^{[1-4]}$ These PCE increases have resulted from the development of 10 new active layer materials, new interfacial layers, optimized device architectures, and 11 optimized active layer morphologies. In particular, in both bulk-heterojunction (BHJ) 12 and bilayer OPVs, control over active layer morphology has widely yielded 13 significant increases in PCE. Through understanding the influence of the bulk and 14 interfacial morphologies, further improvements and more rapid material and device 15 development should be possible. The performance of small-molecule OPVs is 16 believed to depend upon a number of morphological factors, including molecular 17 orientation in the thin film bulk with respect to the substrate normal. ${ }^{[5-11]}$ In small 18 molecule and polymer based OPVs, the exciton diffusion length will depend upon the 19 molecular stacking direction, thus leading to differences in the number of excitons 20 reaching a D-A interface. ${ }^{[12-15]}$ Additionally, the rate of dissociation of these excitons 21 at the interface between electron donating (D) and electron accepting (A) molecules is 22 believed to depend upon the relative orientation of the donor and acceptor molecules 23 at the D-A interface. ${ }^{[6,16]}$ However, recent computational studies of small-molecule 24 interfaces indicate that the molecular orientation of $\mathrm{D}$ molecules with respect to 25 fullerene A molecules can determine the degree of molecular mixing and disorder at 
1 the D-A interface. ${ }^{[17,18]}$ These studies reveal a greater tendency of molecules to

2 intermix when the $\pi$-face of the donor is exposed to the fullerene, i.e. when $\mathrm{D}$

3 molecules are oriented face-on at the D-A interface, than in the edge-on case. The

4 experimental implications of these theoretical predictions have not yet received full

5 attention and a detailed experimental study of the relationship of donor structural

6 order to interfacial mixing is needed. The lack of a precise knowledge of the exact

7 structural configuration at the D-A interface would lead to difficulties in building

8 accurate predictive models and interpretation of device data. Molecular mixing will

9 generally lead to a less crystalline or less aggregated region, which will generally

10 increase the bandgap of the materials in this mixed phase. This mixed interfacial

11 region will then thermodynamically favor exciton dissociation owing to the energetic

12 offsets between the interfacial mixed phase and the pure D and A phases. ${ }^{[19-21]}$ These

13 observations therefore emphasize the intimate relationships between molecular

14 packing, order and interfacial mixing trends that can exist in organic bilayers and in

15 bulk heterojunction thin films. We take the position that the interdependence of these

16 three factors, namely bulk molecular stacking, interfacial molecular orientation and

17 interfacial mixing, should be observable experimentally in devices based on model

18 systems where all three factors can be controlled independently.

19 Unfortunately, the complexity of the bulk heterojunction makes it exceedingly 20 difficult to precisely investigate and decouple the effects of molecular packing 21 orientation from intermixing at the D-A interface. Instead, we opt for model bilayer 22 (BL) systems in which molecular packing orientation and interfacial mixing can be 23 controlled during vacuum deposition as desired. ${ }^{[9,10,22]}$ We first test the premise that 24 face-on molecular packing orientation inherently increases the extent of mixing at the $25 \mathrm{ZnPc}_{6} \mathrm{C}_{60}$ interface in $\mathrm{BL}$ devices. Subsequently, we utilize cryogenic deposition of 
$1 \mathrm{C}_{60}$ on top of $\mathrm{ZnPc}$ to minimize interfacial mixing and compare nominally face-on

2 and edge-on BL devices. We confirm that the extent of molecular mixing is related to

3 the type of molecular orientation present at the D-A interface and that the mixed layer

4 significantly improves the overall device performance by enhancing photocurrent. We

5 go on to show that almost all of the increase in the current with face-on films can be

6 attributed to improved exciton diffusion and to the formation of a spontaneously

7 mixed interface when the donor layer stacking is predominantly face-on.

8

\section{$9 \quad$ Experimental section}

10 Materials. Copper iodide and fullerene- $\mathrm{C}_{60}$ (sublimed grade) were purchased from 11 Sigma Aldrich. Zinc phthalocyanine (triple sublimed) was purchased from Jilin 12 OLED Materials. The materials were degassed in the warm crucible of an Omicron 13 ultra high vacuum (UHV) MBE chamber in the Amassian lab at KAUST overnight 14 before thin film deposition. When devices were made in the Thompson lab at USC, 15 the materials were instead sublimed in-house before use.

16 Photoelectron spectroscopies (UPS and XPS). The ZnPc films prepared for UPS 17 and XPS analyses were deposited on ITO-coated glass substrates (Xinyan 18 Technology) in the UHV MBE chamber and characterized in the XPS/UPS chamber 19 connected directly to the MBE in the Amassian lab at KAUST. The base pressure was $201 \times 10^{-10}$ mbar in the MBE and XPS/UPS chambers. The ZnPc films with thickness of $2120 \mathrm{~nm}$ were deposited at $\sim 0.5 \AA / \mathrm{s}$ with the substrate held at room temperature. The 22 fullerene- $\mathrm{C}_{60}$ overlayer was deposited stepwise. For low thicknesses $(<2 \mathrm{~nm})$ low 23 rates of $0.01-0.02 \AA / \mathrm{s}$ were used whereas a rate of $0.5 \AA / \mathrm{s}$ was used for higher 24 thicknesses $(>2 \mathrm{~nm})$. UPS and XPS spectra of the films were measured with He (I) 
1 excitation $(21.22 \mathrm{eV})$ and monochromated $\mathrm{Al} \mathrm{K \alpha}$ excitation $(1486.7 \mathrm{eV})$, respectively.

2 The Omicron SPHERA hemispherical electron energy analyzer was used to generate

3 the spectra with pass energies of $3.0 \mathrm{eV}$ and $20.0 \mathrm{eV}$ for UPS and XPS, respectively.

4 The dwell time of the samples under UV illumination was minimized to minimize

5 material degradation.

6 Subgap EQE. The measurements were performed in the McGehee lab at Stanford

7 University and in the Amassian lab at KAUST. The EQE spectra were taken at short

8 circuit condition under focused monochromatic illumination from xenon arc

9 (Amassian lab) or tungsten (McGehee lab) lamps. The light beam was modulated by

10 an optical chopper $(275 \mathrm{~Hz})$. The device output current was measured as a function of

11 photon energy using a lock-in amplifier (Stanford Instruments SR 830). The lamp

12 intensity was calibrated with Ge and Si photodiodes.

13 Device fabrication. Devices were fabricated both in the Amassian lab at KAUST and 14 in the Thompson lab at USC. The patterned ITO on glass substrates were cleaned with 15 soap, water, acetone and isopropanol in an ultrasonic bath for 15 minutes for each 16 step followed by a 12-minute UV-ozone exposure. For devices made at KAUST, all 17 organic films were deposited in the Omicron UHV MBE system with a base pressure 18 of $10^{-10} \mathrm{mBar}$ on top of precleaned ITO substrates at rates of $0.5 \AA / \mathrm{s}(\mathrm{ZnPc}), 1 \AA / \mathrm{s}$ $19\left(\mathrm{C}_{60}\right)$ and $0.5 \AA / \mathrm{s}(\mathrm{BCP})$. The CuI layer $(0.2 \AA / \mathrm{s})$ and the $\mathrm{Al}(1 \AA / \mathrm{s})$ electrodes were 20 deposited in a Thermionics vacuum evaporator housed in an Mbraun nitrogen filled 21 glove box. The sample transfer from the UHV to the glove box and back was done via 22 a vacuum suitcase $\left(10^{-7}\right.$ Torr $)$ to avoid air exposure. For samples designed with 23 minimal interfacial mixing, liquid nitrogen cooling of the sample was performed after $24 \mathrm{ZnPc}$ layer deposition and prior to $\mathrm{C}_{60}$ deposition, reducing the substrate temperature 25 to $-100{ }^{\circ} \mathrm{C}$ according to a thermocouple mounted on the sample plate. For samples 
1 requiring a disordered $\mathrm{ZnPc}$ layer and minimal interfacial mixing, the entire bilayer

2 stack was grown after cooling the substrate to $-100{ }^{\circ} \mathrm{C}$. Deliberate interfacial mixing

3 was made possible by co-deposition of a $3 \mathrm{~nm}$ thin mixed $\mathrm{ZnPc}: \mathrm{C}_{60}$ layer (1:1 ratio)

4 on top of the $\mathrm{ZnPc}$ film. The thicknesses of the $\mathrm{ZnPc}$ layer and $\mathrm{C}_{60}$ layer were

5 adjusted to maintain the overall device thickness constant. Devices fabricated in the

6 Thompson lab were prepared at room temperature using a single Angstrom

7 evaporation system for organic and inorganic layers in otherwise similar conditions.

8 Device Characterization. At KAUST, the current-voltage characteristics of the

9 bilayer solar cells were recorded in the nitrogen glove box under AM1.5 simulated

10 illumination (ABET technology) with a spectral irradiance of $100 \mathrm{~mW} / \mathrm{cm}^{2}$. The lamp

11 intensity was calibrated using a reference silicon photodiode. At USC, a Xenon lamp

12 was used instead and the measurements were calibrated to AM 1.5 using the 13 appropriate spectral mismatch.

14 Grazing Incidence Wide Angle $\mathbf{X}$ ray Scattering. GIWAXS measurements were 15 performed at D-line at the Cornell High Energy Synchrotron Source (CHESS) and at 16 the Advanced Light Source (ALS). At CHESS, a $0.5 \mathrm{~mm} \times 0.1 \mathrm{~mm}$ monochromated 17 X-ray beam (double-bounce multilayer monochromator) with $1.15 \AA$ wavelength was 18 used. The diffractograms were recorder using a $50 \times 50 \mathrm{~mm}^{2}$ charged coupling device 19 (CCD) area detector (Medoptics) with pixel size of $46.9 \mu \mathrm{m}$. Experiments were 20 performed at a grazing incidence angle of $0.15^{\circ}$ with respect to the substrate plane.

21 The detector was placed at a distance of $92 \mathrm{~mm}$ from the sample stage. At ALS, 22 experiments were performed with a monochromated X-ray beam (1.24 A wavelength) 23 and a fast 2D detector (PILATUS 1M from Dectris) with pixel size of $172 \mu \mathrm{m}$. A 24 shallow angle of $0.15 \square$ was chosen for the incident $\mathrm{x}$-rays with respect to the sample 25 plane and the detector was placed at a distance of $302.68 \mathrm{~mm}$ from the sample stage. 
1 Atomic Force Microscopy. The surface morphology of $\mathrm{ZnPc}$ films was examined

2 using tapping mode AFM (Agilent 5400).

3 UV-Vis Absorption. Absorption spectra were acquired with a Cary 5000 (Varian)

4 spectrophotometer on $\mathrm{ZnPc}$ films prepared on ITO-coated glass in the same 5 conditions used for device fabrication.

6

\section{$7 \quad$ Results and discussion}

8 Spontaneous interfacial mixing in edge-on and face-on packed $\mathrm{ZnPc} / \mathrm{C}_{60}$ bilayer 9 films

10 We begin by investigating intermixing at the $\mathrm{ZnPc}^{-\mathrm{C}_{60}}$ interface and the texture of $11 \mathrm{ZnPc}$ thin films. The molecular packing texture was modified from predominantly 12 edge-on texture on $\mathrm{ITO} / \mathrm{SiO}_{2}$ to face-on texture by pre-coating the substrate with a 2 $13 \mathrm{~nm}$ templating layer of copper iodide $(\mathrm{CuI}) .{ }^{[6,10]}$ In Figure 1, we present the two14 dimensional grazing incidence wide angle x-ray scattering (GIWAXS) data and 15 corresponding integrated intensities in the horizontal (in-plane) and vertical (out-of16 plane) directions, which help us confirm the crystalline texture and the stacking 17 orientation of $\mathrm{ZnPc}$ in thin films. ${ }^{[6,10,23]}$ The ZnPc/ITO film exhibits scattering peaks 18 at $4.95 \mathrm{~nm}^{-1}$ in the vertical direction and $5.11 \mathrm{~nm}^{-1}$ and $18.52 \mathrm{~nm}^{-1}$ in the horizontal 19 direction, corresponding respectively to $\mathrm{d}_{200}=1.27 \mathrm{~nm}, \mathrm{~d}_{002}=1.23 \mathrm{~nm}$ and $\mathrm{d}_{010}=0.34$ $20 \mathrm{~nm}$ of the $\gamma$ phase of $\mathrm{ZnPc}$ with the (002) plane parallel to the substrate, indicative of 21 the edge-on stacking texture. By contrast, the $\mathrm{ZnPc} / \mathrm{CuI} / \mathrm{ITO}$ films exhibit scattering 22 peaks at $4.91 \mathrm{~nm}^{-1}$ and $18.96 \mathrm{~nm}^{-1}$ in the vertical direction and $4.76 \mathrm{~nm}^{-1}$ in the 23 horizontal direction, corresponding respectively to $\mathrm{d}_{002}=1.28 \mathrm{~nm}, \mathrm{~d}_{010}=0.33 \mathrm{~nm}$ and $24 \mathrm{~d}_{200}=1.32 \mathrm{~nm}$ of the $\gamma$ phase of $\mathrm{ZnPc}$ with the (010) plane parallel to the substrate, 
1 indicative of the face-on stacking texture. The (010) peak corresponds to scattering

2 along the $\pi-\pi^{*}$ stacking direction, and clearly orients itself parallel to the substrate

3 plane in edge-on stacked molecules and perpendicular to the substrate plane in face-

4 on stacked molecules. We found these results to be consistent with what has been

5 reported previously. ${ }^{[6,23]}$

6 We utilize photoelectron spectroscopy to monitor the attenuation of the ZnPc signal

7 during stepwise deposition of $\mathrm{C}_{60}$ on top of the pristine $\mathrm{ZnPc}$ films. X-ray

8 photoelectron spectroscopy (XPS) and ultraviolet photoelectron spectroscopy (UPS)

9 are non-invasive and do not induce further intermixing, as sputtering based depth-

10 profiling techniques such as secondary ion mass spectrometry may. In UPS and XPS

11 the escape probability of photoelectrons decreases exponentially with the sampling

12 depth. This feature can be used to probe intermolecular mixing at the interface

13 between two layers. In the case of a non-mixing, discrete interface, the signal from the

14 underlayer follows a rapid exponential decay as it gets buried, with typical inelastic

15 mean free paths of $c a .1 \mathrm{~nm}$ in UPS and $c a .3 \mathrm{~nm}$ in XPS. ${ }^{[24,25]}$ If mixing occurs, the

16 rate of attenuation from the underlayer will decrease as $\mathrm{ZnPc}$ mixes with the top $\mathrm{C}_{60}$

17 layer. We perform stepwise deposition of $\mathrm{C}_{60}$ on top of a pristine $\mathrm{ZnPc}$ film at room

18 temperature (RT) while monitoring the nitrogen core electron $\mathrm{N}$ 1s signal (XPS) or

19 the ZnPc HOMO peak (UPS) area (Figure 2). The observed trends in the decay rates

20 reveal a slower decrease in the case of face-on $\mathrm{ZnPc}$ as opposed to edge-on $\mathrm{ZnPc}$.

21 These differences in attenuation rates most likely indicate more interfacial molecular

22 mixing taking place in the case of face-on $\mathrm{ZnPc}$ on ITO/CuI than in the case of edge-

23 on $\mathrm{ZnPc}$ on ITO. Since differences in film morphology and surface coverage would

24 invalidate the in situ XPS and UPS conclusions, atomic force microscopy (AFM) was

25 performed on the edge-on and face-on $\mathrm{ZnPc}$ films before and after $\mathrm{C}_{60}$ deposition to 
1 compare the roughness of the bare films and surface coverage with $\mathrm{C}_{60}$. The AFM

2 analysis indicates that both $\mathrm{ZnPc}$ films are initially equally smooth and become

3 equally smoother after $3 \mathrm{~nm} \quad \mathrm{C}_{60}$ deposition (Figure S1, Supplementary

4 Information). This confirms that the differences in attenuation trends are not

5 primarily influenced by morphological factors and can be assigned to differences in

6 the extent of interfacial mixing. To further validate these observations, we have

7 complimented the surface-sensitive in situ XPS and UPS studies with more bulk

8 sensitive measurements relying on a systematic GIWAXS analysis of fullerene-

9 fullerene scattering with increasing thickness of fullerene on face-on and edge-on 10 stacked ZnPc. Above the critical angle of the film, GIWAXS probes the entire sample

11 volume, including the buried fullerene-ZnPc interface. We specifically look for the 12 evolution of fullerene scattering features with increasing deposited thickness, which 13 should behave monotonically if fullerene does not mix with the underlayer, whereas 14 the same signal should decrease if instead fullerene diffuses and mixes with the ZnPc 15 underlayer. While no significant change was detected in the evolution of the ZnPc 16 peak, the $\mathrm{C}_{60}$ peak evolution indicates meaningful differences between edge-on and 17 face-on $\mathrm{ZnPc}_{\mathrm{C}} \mathrm{C}_{60}$ bilayers (Figure S2, Supplementary Information). Fitting the 18 fullerene peak to a Gaussian function and preforming appropriate background 19 subtractions, we plot the integrated fullerene signal as a function of the layer 20 thickness (Figure S3, Supplementary Information). We observe that the $\mathrm{C}_{60}$ signal 21 increases quasi linearly both on face-on and edge-on $\mathrm{ZnPc}$ films with increased 22 fullerene thickness but consistently, the fullerene signal from edge-on samples are 23 higher than that of face-on samples. This shows that in the face-on case, a portion of 24 the $\mathrm{C}_{60}$ layer, and particularly the part deposited initially, scatters less than on edge-on 25 ZnPc. Yet, it is known that the $\mathrm{C}_{60}$ peak has the same characteristics in both cases 
1 meaning the crystallite orientation in both cases were isotropic and the d-spacing is

2 identical within the experimental error. We further remark that, the integrated

3 scattered intensity of the $30 \mathrm{~nm}$ thick fullerene film on edge-on $\mathrm{ZnPc}$ is remarkably

4 close to that of $30 \mathrm{~nm} \mathrm{C}_{60}$ on bare ITO while the scattered intensity of the $30 \mathrm{~nm}$ thick

5 film on face-on $\mathrm{ZnPc}$ is substantially lower than the control on ITO, indicating that

6 more $\mathrm{C}_{60}$ was lost through diffusion into the underlayer (Figure S2, Supplementary

7 Information). In light of the GIWAXS, XPS and UPS results, we conclude that when

8 fullerene- $\mathrm{C}_{60}$ is deposited on face-on $\mathrm{ZnPc}$, it mixes to a greater extent than when it is

9 deposited on edge-on $\mathrm{ZnPc}$, in agreement with computational predictions which have

10 identified differences in surface energies of face-on and edge-on films as the main

11 driving force for interfacial mixing. ${ }^{[17]}$

\section{Impact of interfacial mixing on the photovoltaic performance}

13 We evaluate the impact of spontaneous interfacial mixing for the above molecular 14 packing orientations by fabricating a series of devices with different $\mathrm{ZnPc}$ molecular 15 packing orientations at room temperature. The device architectures are based on the 16 standard device stacks: ITO/ZnPc $(20 \mathrm{~nm}) / C_{60}(45 \mathrm{~nm}) / B C P(12 \mathrm{~nm}) / \mathrm{Al}(100 \mathrm{~nm})$ for 17 edge-on $\mathrm{ZnPc}$ and ITO/CuI $(2 \mathrm{~nm}) / \mathrm{ZnPc}(20 \mathrm{~nm}) / \mathrm{C}_{60}(45 \mathrm{~nm}) / \mathrm{BCP}(12 \mathrm{~nm}) / \mathrm{Al}(100$ $18 \mathrm{~nm}$ ) for face-on $\mathrm{ZnPc}$. The $\mathrm{J}-\mathrm{V}$ curves and associated bilayer cartoons are presented in 19 Figure 3, while device parameters and statistics are summarized in Table 1. The 20 device characteristics of room temperature $\mathrm{ZnPc}$ and $\mathrm{C}_{60}$ on ITO and $\mathrm{CuI} / \mathrm{ITO}$ are 21 consistent with previously reported data whereby an increase in PCE of $\sim 70 \%$ was 22 observed, ${ }^{[4]}$ namely from $1.7 \%$ to $2.9 \%$ in going from a predominantly edge-on to 23 face-on stacking orientation in the $\mathrm{ZnPc}$ layer. 

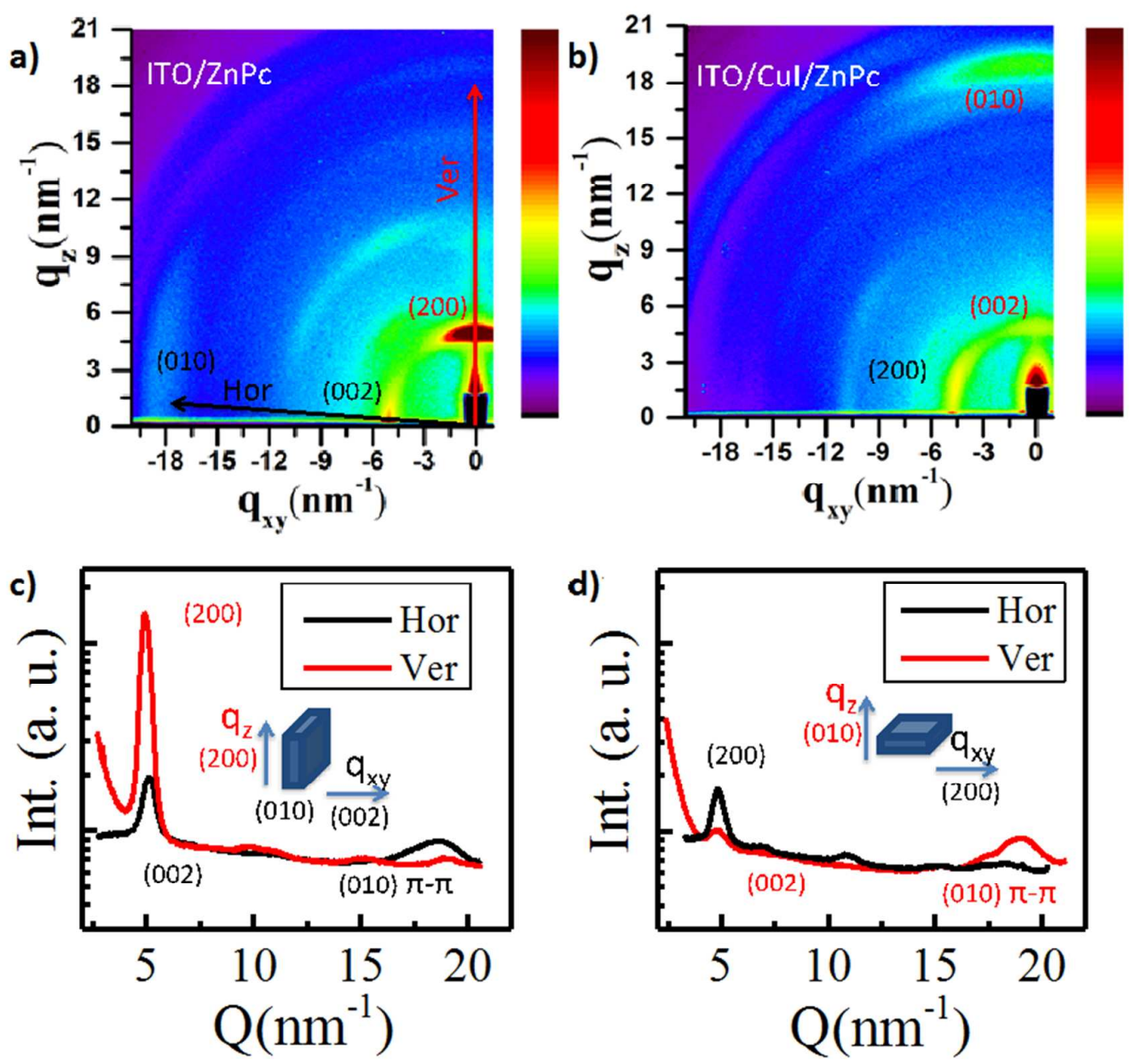

2 Figure 1. (a, b) 2D GIWAXS patterns of $20 \mathrm{~nm}$-thick and (c, d) integration plots in 3 horizontal (black) and vertical (red) of the $\mathrm{ZnPc}$ films grown on $(\mathbf{a}, \mathbf{c}) \mathrm{ITO} /$ glass and 4 (b, d) on $2 \mathrm{~nm}$ CuI-coated ITO/glass substrates. $\mathrm{Q}_{\mathrm{xy}}$ and $\mathrm{Q}_{\mathrm{z}}$ refer to the in-plane and 5 out-of-plane components of the scattering vector, respectively. 6
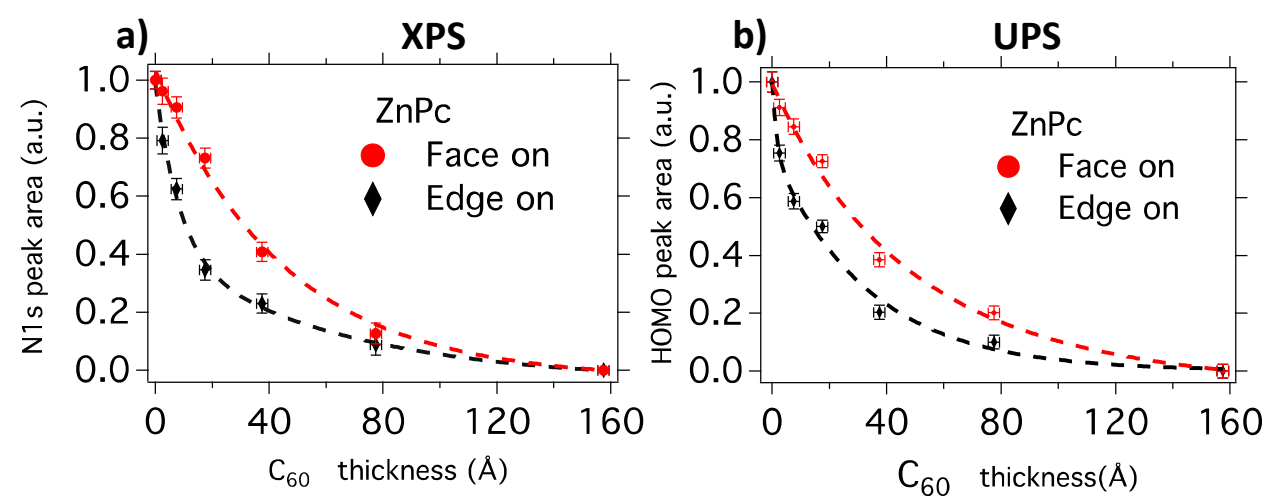

Figure 2. Attenuation of the nitrogen 1s peak from XPS (a) and $\mathrm{ZnPc}$ HOMO peak

9 from UPS (b) of face-on and edge-on $\mathrm{ZnPc}$ with increasing $\mathrm{C}_{60}$ thickness. Both curves

10 feature a more rapid decay of the photoelectron signal from the $\mathrm{ZnPc}$ layer in the

11 edge-on case. In the face-on case the slower decay of the signal suggests more 
1 interfacial mixing. The dotted lines are added as visual guides to the eye. The nitrogen

$21 \mathrm{~s}$ peak was selected for the analysis because of its larger peak strength as compared

3 to the $\mathrm{Zn} 2 \mathrm{p}$ peak.

4

5 Table 1. Photovoltaic performance characteristics measured under $100 \mathrm{~mW} / \mathrm{cm}^{2}$

6 simulated AM1.5 irradiation. $\mathrm{J}_{\mathrm{sc}}$ is short-circuit current density, FF is fill factor, $\mathrm{V}_{\mathrm{oc}}$ is

7 the open-circuit voltage and PCE is power conversion efficiency. Standard bilayer

8 refers to the RT devices under investigation.

\begin{tabular}{|c|c|c|c|c|c|c|c|c|}
\hline \multirow{2}{*}{$\begin{array}{c}\text { Description } \\
\begin{array}{c}\text { ZnPc stacking } \\
\text { orientation }\end{array}\end{array}$} & \multicolumn{2}{|c|}{ Standard bilayer } & \multicolumn{2}{|c|}{$\begin{array}{l}\text { "Frozen" } C_{60} \\
\text { (More abrupt } \\
\text { interface) }\end{array}$} & \multicolumn{2}{|c|}{$\begin{array}{l}\text { Standard bilayer with } \\
\text { mixed interface }\end{array}$} & \multicolumn{2}{|c|}{$\begin{array}{l}\text { "Frozen" } \mathrm{ZnPc} \text { and } \mathrm{C}_{60} \\
\text { (Disordered bulk and } \\
\text { more abrupt interface) }\end{array}$} \\
\hline & Edge-on & Face-on & Edge-on & Face-on & Edge-on & Face-on & Edge-on & Face-on \\
\hline ZnPc dep. temp. $\left[{ }^{\circ} \mathrm{C}\right]$ & 25 & 25 & 25 & 25 & 25 & 25 & -100 & -100 \\
\hline $\mathrm{C}_{60}$ dep. temp. $\left[{ }^{\circ} \mathrm{C}\right]$ & 25 & 25 & -100 & -100 & 25 & 25 & -100 & -100 \\
\hline Mixed interlayer? & No & No & No & No & Yes & Yes & No & No \\
\hline Voc $[\mathrm{V}]$ & $0.57 \pm 0.02$ & $0.61 \pm 0.01$ & $0.56 \pm 0.03$ & $0.61 \pm 0.02$ & $0.60 \pm 0.02$ & $0.60 \pm 0.02$ & $0.58 \pm 0.02$ & $0.58 \pm 0.01$ \\
\hline $\mathrm{FF}[\%]$ & $63.30 \pm 0.5$ & $70 \pm 0.4$ & $59.70 \pm 0.5$ & $68.10 \pm 0.6$ & $62.40 \pm 0.6$ & $69.10 \pm 0.3$ & $66.30 \pm 0.4$ & $64.00 \pm 0.6$ \\
\hline $\mathrm{J}_{\mathrm{sc}}\left[\mathrm{mA} / \mathrm{cm}^{2}\right]$ & $4.70 \pm 0.1$ & $6.70 \pm 0.1$ & $4.10 \pm 0.1$ & $5.60 \pm 0.1$ & $5.60 \pm 0.1$ & $7.70 \pm 0.1$ & $4.40 \pm 0.1$ & $5.20 \pm 0.1$ \\
\hline PCE [\%] & $1.70 \pm 0.06$ & $2.90 \pm 0.04$ & $1.40 \pm 0.04$ & $2.30 \pm 0.05$ & $2.10 \pm 0.05$ & $3.20 \pm 0.04$ & $1.70 \pm 0.05$ & $1.90 \pm 0.06$ \\
\hline
\end{tabular}

10

11

12

13 ZnPc (Table 1: "Frozen" $\mathrm{C}_{60}$, Figure 3a, b). With these cells, a reduction in the device

14 PV performance is observed from $1.7 \%$ to $1.4 \%$ and from $2.9 \%$ to $2.3 \%$ in the edge-

15 on and face-on cases, respectively. This mostly stems from a reduction in

16 photocurrents; $\sim 0.5 \mathrm{mAcm}^{-2}$ vs $\sim 1.10 \mathrm{mAcm}^{-2}$ drops in the edge-on and face-on cases,

17 respectively. A small decrease in FF is also noted in both cases and could be

18 attributed to the presence of more disorder in the $\mathrm{C}_{60}$ layer deposited in cryogenic

19 conditions. While we do not exclude the possibility of some interfacial mixing

20 occurring once the device returns slowly to room temperature (Figure S4,

21 Supplementary Information), we expect that molecules diffuse less once the bilayer

22 is formed since molecules in the bulk or at the interface often do not enjoy the same

23 degree of mobility as do molecules at surfaces. ${ }^{[26-28]}$ The significant reduction in $\mathrm{J}_{\mathrm{sc}}$

24 upon minimizing the intermixing suggests that the difference in photocurrents in 
1 devices prepared at room temperature is in part due to differences in the degree of

2 interfacial mixing for face-on and edge-on packed $\mathrm{ZnPc}$ films. This more favorably

3 affects the BL device photocurrent in the face-on case since the mixed phase has been

4 shown to induce band gap widening which leads to an energetic cascade that assists

5 excitons dissociation. ${ }^{[21,33]}$

6

a)

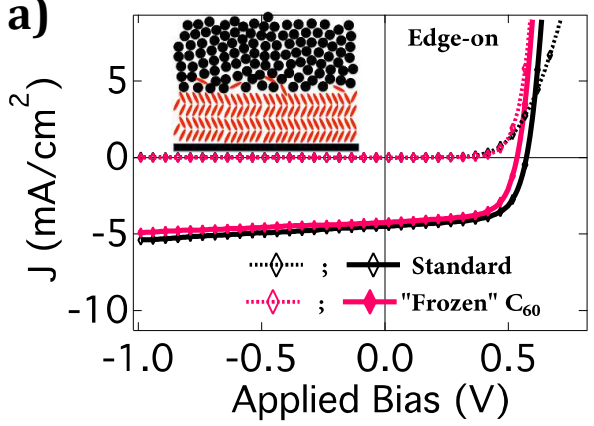

c)

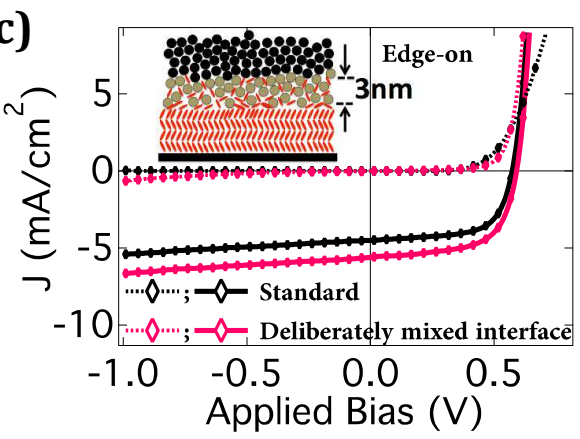

e)

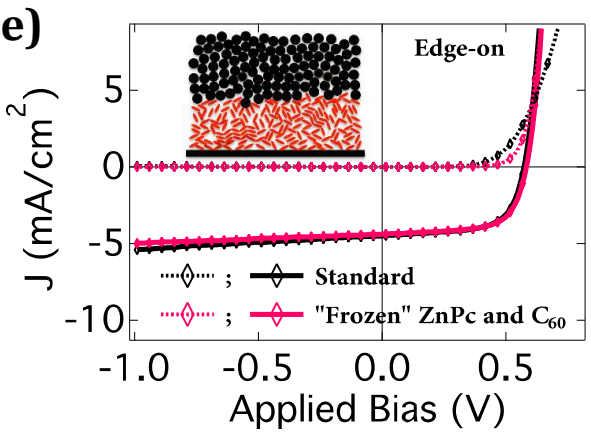

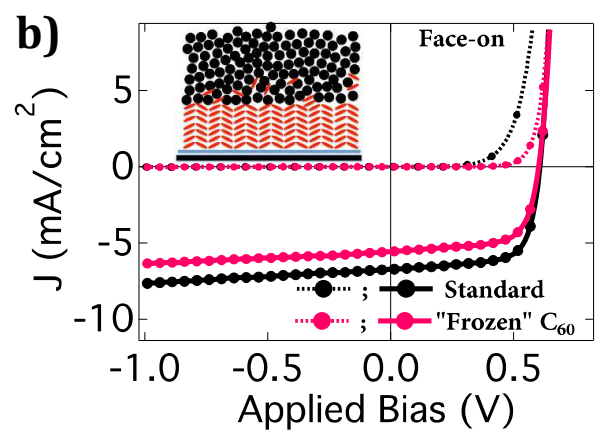
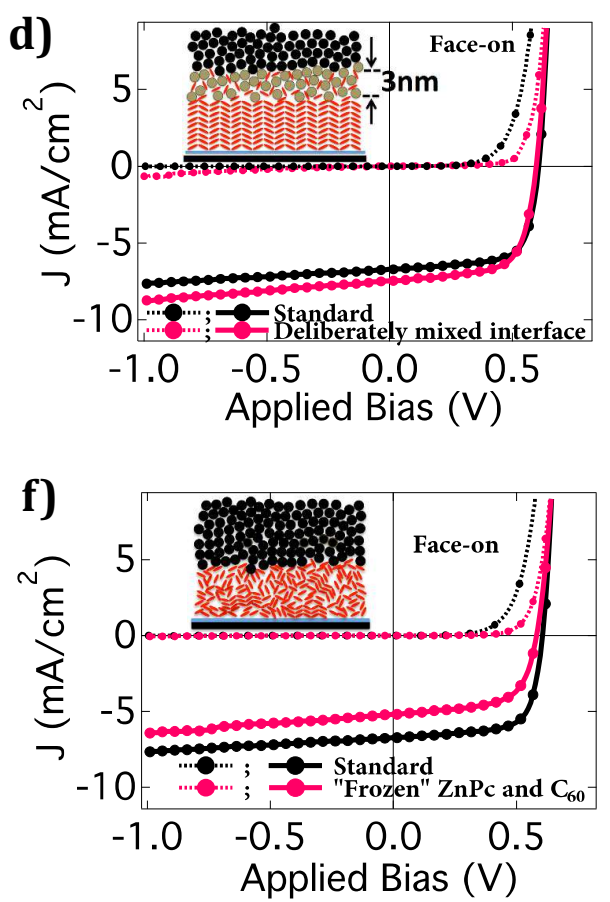

Figure 3. Current-voltage characteristics under illumination (solid curves) and in the dark (dotted lines) of face-on and edge-on $\mathrm{ZnPc} / \mathrm{C}_{60} \mathrm{BL}$ solar cells with altered interfacial and bulk molecular packing structures. The black color shows the characteristics of the standard devices for comparison. The inset schematically illustrates the $\mathrm{ZnPc}$ molecular conformation and the extent of mixing at the interface. The grey circles illustrate fullerene- $\mathrm{C}_{60}$ in the deliberately mixed layer $(\mathbf{a}, \mathbf{b})$ : The 
1 fullerene- $\mathrm{C}_{60}$ is deposited at $-100{ }^{\circ} \mathrm{C}$ (referred to as "frozen") to reduce interfacial 2 mixing in order to achieve a more abrupt interface. (c, d): Devices made with a 3 deliberately mixed co-deposited interfacial layer demonstrating the impact of the 4 mixed layer on PV performance. (e, $\mathbf{f}$ ): Both the $\mathrm{ZnPc}$ and $\mathrm{C}_{60}$ layers are deposited at $5-100{ }^{\circ} \mathrm{C}$ and therefore "frozen" to achieve a more abrupt interface and a disordered $6 \quad$ ZnPc bulk.

To further probe whether interfacial mixing is a key and positive contributor to the

9 performance improvement in face-on vs. edge-on BL solar cells, BL devices with a deliberately 1:1 mixed $3 \mathrm{~nm}$ interfacial layer on top of edge-on and face-on $\mathrm{ZnPc}$

11 films (Figure 3c, d) were fabricated. As expected, the presence of the mixed layer 12 improves the edge-on BL device efficiency by $\sim 23 \%$ from $1.7 \%$ to $2.1 \%$, and by $13 \sim 10 \%$ in the face-on case. The deliberately introduced mixed layer is hence less 14 beneficial for the face-on device, which already had a mixed region spontaneously 15 formed. More importantly the $\mathrm{V}_{\text {oc }}$ of the face-on device remains unchanged at $\sim 0.6 \mathrm{~V}$ 16 while an increase in $\mathrm{V}_{\mathrm{oc}}$, to practically an identical value to that of the face-on device, 17 is observed for the edge-on device. In the "frozen" $\mathrm{ZnPc}$ devices (Table 1) the opposite trend in $\mathrm{V}_{\mathrm{oc}}$ is observed and vice versa, confirming that the reason for the observed changes in Voc is associated to the degree of interfacial mixing. Although a 20 more focused study of recombination mechanisms is needed to fully understand these 21 changes in $\mathrm{V}_{\mathrm{oc}}$, the observations presented herein provide strong indication that the 22 spontaneously formed mixed layer is responsible for the higher $\mathrm{V}_{\mathrm{oc}}$ observed in the 23 face-on devices. However, this increase in $\mathrm{V}_{\mathrm{oc}}$ stemming from the mixed phase is $a$ 24 priori counterintuitive when examined from the point of view of disorder in the mixed 25 interfacial layer, but as will be discussed later and has been shown in the context of 26 polymer:fullerene solar cells, disorder in the mixed interfacial phase favorably 27 impacts interfacial energetics in a manner that favors charge generation. ${ }^{[21,33]}$ Hence, 28 in light of these results together with the photoelectron spectroscopy data, it appears 
1 that more spontaneous interfacial mixing occurs in nominally face-on BL devices than

2 in edge-on ones fabricated at RT, and that this interfacial mixing is in part responsible

3 for the higher photocurrent, $\mathrm{V}_{\mathrm{oc}}$ and power conversion efficiency in the face-on

4 devices.

5 A direct and inevitable consequence of intermixing should be an increase in the 6 number of $\mathrm{ZnPc}_{\mathrm{C}} \mathrm{C}_{60}$ bimolecular charge transfer $(\mathrm{CT})$ complexes at the $\mathrm{D}-\mathrm{A}$ interface.

7 An important characteristic of the CT complex is that it can be photoexcited by direct

8 sub-gap monochromatic illumination and the resulting excited states can generate free

9 charges which can be collected by the electrodes. The generated charges can be 10 detected by a sensitive external quantum efficiency (EQE) measurement performed 11 on a PV device, making it possible to directly investigate interfacial mixing in actual 12 devices. The EQE from the charge transfer state absorption band is modeled by 13 equation 1.

$$
E Q E=\frac{f}{E \sqrt{4 \pi \lambda k T}} \exp \left(\frac{-\left(E_{C T}+\lambda-E\right)^{2}}{4 \lambda k T}\right)
$$

15 Here, $\mathrm{E}_{\mathrm{CT}}$ is the energy of the $\mathrm{CT}$ state, $\lambda$ is related to the reorganization energy and 16 energetic disorder, ${ }^{[29]}$ and $f$ is directly related to the intensity of the CT absorbance 17 band. Specifically, $f$ is the product of the number of interfacial CT complexes, the 18 internal quantum efficiency (IQE) of the CT state, and the square of the electronic 19 coupling matrix element. Therefore, $f$ should be proportional to the film thickness if 20 the CT states are assumed distributed evenly throughout the film. In addition, with $f$ 21 being directly proportional to the number of CT complexes, an increase in $f$ for the 22 same material system can be utilized as a means of determining the relative number of 23 CT complexes. Based on this, we have fabricated a series of BL devices with variable 24 ZnPc thickness and measured the strength of the CT absorbance (Figure 4a,b). 
1 Fitting the $\mathrm{CT}$ band to the above equation, we extract $f$ and $\mathrm{E}_{\mathrm{CT}}$ for each device (see

2 Table S1, Supplementary Information). The magnitude of the CT absorbance

3 increases with $\mathrm{ZnPc}$ thickness (Figure $4 \mathrm{~b}$, inset) in the face-on case ( $90 \%$ increase in

4 f) while remaining virtually unchanged in the edge-on case. It is important to note that

5 the direct excitation $(1.5-2.0 \mathrm{eV})$ of the face-on $\mathrm{ZnPc}$ also increases with increased

6 thickness and follows the same trend as the CT band excitation. This could therefore

7 lead to a misinterpretation of the observed increase in $f$ (Figure $4 \mathbf{b}, \mathbf{d}$ ). However,

8 looking at the corresponding trends in the edge-on $\mathrm{ZnPc}$ whereby the increase in the

9 direct excitation leads to virtually no increase in the CT band excitation (Figure 4a,c),

10 we conclude that the opposite trend observed in the face-on case is indeed due to a

11 real increase in $\mathrm{CT}$ absorption at the D-A interface and not to any tailing of the

12 absorption of ZnPc. In addition, we rule out the possibility that the difference in CT

13 state absorption may be due to differences in interfacial physical roughness between

14 the edge-on and face-on films on the basis of AFM analysis (Figure S1,

15 Supplementary Information), which revealed very similar surface roughness for

16 both types of films. Similarly, one might argue that the D/A electronic coupling

17 should be lower in the edge-on orientation, which decreases $f$, resulting in fewer

18 subgap states being excited upon illumination. However, we believe that even if that

19 were the case, the dependence of the CT absorption on the film thickness for films

20 prepared in identical conditions should still be representative of the variation in the

21 true concentration of CT states that are formed as the film thickness is varied, making

22 such relative comparisons meaningful. Hence, the increase in CT absorbance most

23 likely corresponds to an increase in the number of CT states as a result of increased

24 mixing at the face-on $\mathrm{ZnPc} / \mathrm{C}_{60}$ interface. The fact that $f$ increases with film thickness

25 in case of face-on stacked $\mathrm{ZnPc}$ films, but not for edge-on stacked films is consistent 
1 with no additional intermixing occurring upon deposition of the first monolayer of

2 fullerene in the latter case, whereas in the former case the supply of more fullerene

3 allows the system the undergo further intermixing, resulting in more CT state

4 formation.

5

6

7 Role of bulk molecular packing and interfacial mixing on the amount of excitons

8

9 We now have a better handle over the influence that the degree of mixing at the D-

10 A interface can have on the device photocurrent. One should not forget, however, that

11 differences in molecular orientation at the interface stem from stacking differences in

12 the bulk of the donor film. Bulk orientation differences may impact the exciton

13 diffusion length and film absorbance, and therefore affect the device photocurrent.

14 We evaluate the differences in the number of excitons reaching a D-A interface by 15 comparing the $\mathrm{EQE}$ of $\mathrm{BL}$ devices with different $\mathrm{ZnPc}$ thickness, both in 16 predominantly edge-on and face-on orientations (Figure 4c,d). The EQE signal in the 17 case of the edge-on BL devices increases marginally with the ZnPc layer thickness in 18 the key spectral range 1.5-2.2 eV corresponding to the $\mathrm{ZnPc}$ Q-band absorption. By 19 contrast, a significant increase of EQE is seen with increasing thickness of face-on 20 ZnPc. These observations indicate that more excitons are reaching the D-A interface 21 for thicker $\mathrm{ZnPc}$ films in the presence of predominantly face-on stacking of $\mathrm{ZnPc}$ 22 molecules in the donor layer than in the presence of edge-on stacking, likely due to 23 both differences in exciton diffusion length and interfacial mixing in favor of the 24 former. 
a)

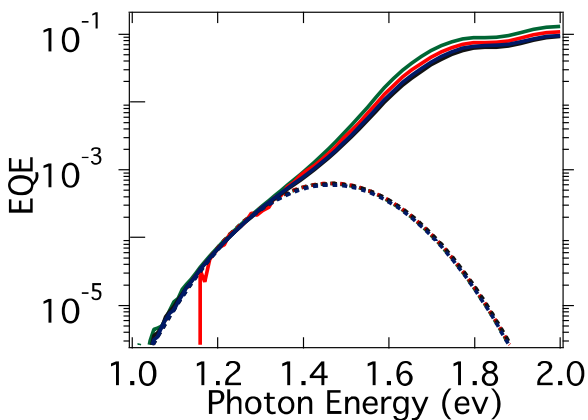

c)

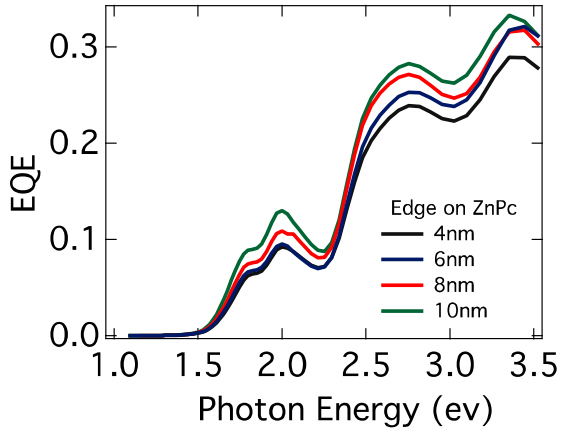

\section{b)}
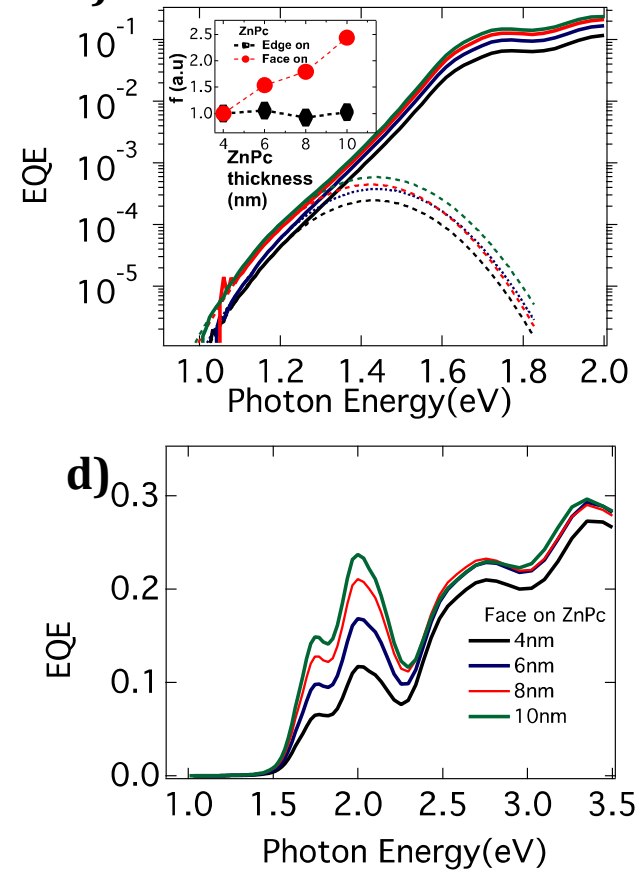

Figure 4. Top: Spectrally resolved sensitive $\mathrm{EQE}$ spectra of $\mathrm{ZnPc} / \mathrm{C}_{60} \mathrm{BL}$ cells including in the subgap spectral range with varying thickness of predominantly edgeon (a) and face-on (b) stacked $\mathrm{ZnPc}$ deposited at room temperature showing the evolution of the CT absorption band. The fits (dotted curves) are performed to extract the electronic coupling term $(f)$, which is directly proportional to the number of interfacial charge transfer states. Inset of (b): Evolution of $f$ with $\mathrm{ZnPc}$ film thickness for the edge-on and face-on stacked BL devices. The increase observed in the case of face-on stacked $\mathrm{ZnPc}$ indicates increased degree of interfacial mixing. Bottom: Evolution of the spectral response of the $\mathrm{ZnPc} / \mathrm{C}_{60} \mathrm{BL}$ solar cells with edge-on (c) and face-on $\mathrm{ZnPc}$ donor layer (d) as a function of the $\mathrm{ZnPc}$ layer thickness. For the edgeon $\mathrm{ZnPc} \mathrm{BL}$ devices, the $\mathrm{EQE}$ increases only marginally with increasing $\mathrm{ZnPc}$ layer thickness. For the face-on $\mathrm{ZnPc} \mathrm{BL}$ devices, the EQE signal increases more markedly with increasing $\mathrm{ZnPc}$ thickness.

\section{We further seek to isolate and validate the contributions to the photocurrent from} the bulk molecular packing and interfacial mixing. We have fabricated devices wherein both the $\mathrm{ZnPc}$ and the $\mathrm{C}_{60}$ layers are deposited at cryogenic temperatures. This promotes a reduced molecular ordering in the $\mathrm{ZnPc}$ bulk (Figure S5, Supplementary Information) while also maintaining a minimally mixed interface.

The device results (Table 1, "Frozen" $\mathrm{ZnPc}$ and $\mathrm{C}_{60}$ and Figure 3e,f) show very close 
1 PCEs for BL devices on ITO and ITO/CuI, namely $1.7 \%$ and $1.9 \%$, respectively,

2 consistent with the fact that both the interface and the bulk are nearly identical in the

3 two device architectures. In comparison to the previous case in which $\mathrm{C}_{60}$ was

4 deposited under cryogenic conditions on $\mathrm{ZnPc}$ deposited at room temperature (2.3\%),

5 the BL device fabricated fully in cryogenic conditions on CuI/ITO exhibits a further

6 decrease in PCE attributed to all device performance parameters degrading, especially

7 the FF. We find it intriguing and telling that a disordered crystalline $\mathrm{ZnPc}$ film should

8 behave just as well as a predominantly edge-on oriented polycrystalline film in terms

9 of device performance. This may be an indication that out-of-plane exciton diffusion

10 in small-molecule donor films exhibiting predominantly in-plane $\pi$-stacking is similar

11 to that of a fully disordered layer. Importantly, these results indicate that bulk

12 ordering and stacking direction play important roles alongside interfacial mixing at

13 improving PV performance in small-molecule organic solar cells. The same may not

14 be true in polymer-fullerene solar cells, where intrachain transport is believed to be a

15 dominant contributor to carrier and energy transport. ${ }^{[30-32]}$ Finally, the mixed

16 interfacial layer in face-on devices is believed to introduce an energetic gradient

17 between the pure fullerene and $\mathrm{ZnPc}$ phases that should assist in charge separation

18 and reduce geminate recombination as has been demonstrated by others. ${ }^{[21,33]}$ This

19 could explain the increase in $\mathrm{V}_{\mathrm{oc}}$ in the edge-on $\mathrm{ZnPc}$ device with deliberate mixing.

20 However a full explanation requires a detailed investigation and is outside the scope

21 of this study.

\section{Conclusion}

24 The present study reveals the impact that stacking orientation can have on the 25 donor-acceptor interface in organic solar cells. We have focused our attention on 
1 vacuum-deposited bilayer solar cells where state-of-the-art ultra high vacuum

2 molecular beam deposition techniques allow us to control and ultimately decouple

3 interfacial and bulk molecular stacking effects, with broad implications for either

4 solution and vacuum deposited small-molecule, oligomer and polymer based bilayer

5 and bulk heterojunction solar cells.

6 Through careful experimentation we find that differences in stacking orientation in

7 the bulk and surface of $\mathrm{ZnPc}$ films can lead to significant differences in the mixing

8 behavior at the interface with $\mathrm{C}_{60}$. We demonstrate that spontaneous interfacial

9 mixing at room temperature is more favored in face-on terminated $\mathrm{ZnPc}$ films than in

10 edge-on terminated ones. The induced mixed layer benefits the bilayer solar cells by

11 increasing the photocurrent and open circuit voltage and its presence at the D-A

12 interface is believed to promote charge separation owing to changes in the energetic

13 landscape that it creates. We have also found that when the molecules pack face-on in

14 the bulk, this contributes to more excitons reaching D-A interfaces, and hence also

15 accounts for the observed improvement in device photocurrent.

16 This study highlights the importance of molecular packing in OPV bilayer devices

17 and its dual impact on both bulk and interfacial PV processes. The ability to precisely

18 control the molecular orientation both in the bulk and at the D-A interface in order to

19 achieve more face-on donor films may play a key role in enabling further

20 improvements in PV performance. This study equally highlights the risks associated

21 to extrapolating interface-related conclusions and interpreting device performances

22 simply on the basis of bulk molecular stacking information in small molecules solar

23 cells either in bilayer or bulk heterojunction device configurations without looking at

24 the state of frontier molecules before and after bilayer formation. 
1 While some of the outcomes of the study, such as the link between bulk molecular

2 stacking and exciton diffusion, may not directly apply beyond bilayer solar cells to

3 modern bulk heterojunction systems, our approach nevertheless offers opportunities

4 to investigate a subset of interfaces within a typical bulk heterojunction solar cell but

5 which, due to their extremely complex combination of morphologies, currently cannot

6 be directly addressed using mean-field spectroscopic techniques. Certainly, the

7 conclusions of this study, with regards to spontaneous interfacial mixing, its link to

8 conformation of frontier molecules and the influence of mixing on free carrier

9 generation and recombination are believed to be widely applicable to bulk

10 heterojunction solar cells. ${ }^{[35-37]}$

\section{Supporting Information Available}

13 AFM topographic images of pure $\mathrm{ZnPc}$ and $\mathrm{ZnPc} / \mathrm{C}_{60}$ bilayers films; GIWAXS data 14 for $\mathrm{ZnPc} / \mathrm{C}_{60}$ bilayers and grown frozen $\mathrm{ZnPc}$ films; XPS attenuation curves for $15 \mathrm{ZnPc}_{\mathrm{C}} \mathrm{C}_{60}$ bilayers with cryogenically cooled substrate (abrupt interface) and 16 deliberately mixed interface; EQE spectra for standard, mixed interface, abrupt 17 interface and $\mathrm{ZnPc}$ with reduced crystallinity bilayer devices; electronic coupling 18 term and charge transfer state energy values for $\mathrm{ZnPc} / \mathrm{C}_{60}$ bilayers.

20 This information is available free of charge via the Internet at http://pubs.acs.org/.

21

\section{Author Information}

\section{Corresponding Author}

24 * (A.A)-E-mail: aram.amassian@kaust.edu.sa 
2

3

4

5

6

7

8

9

10

\section{Acknowledgements}

2 This work was supported by the Office of Competitive Research Funds at the King Abdullah

3 University of Science and Technology. Portions of this work were done at the Center of 4 Advanced Molecular Photovoltaics, at the Stanford Synchrotron Radiation, at the Cornell 5 High Energy Synchrotron Source (CHESS) and at the Advanced Light Source (ALS). 6 G.O.N.N., K.R.G., M.D.M., and A.A. acknowledge the Office of Competitive Research 7 Funds for a GRP-CF award. K.R.G. and A.A. acknowledge SABIC for a post-doctoral 8 fellowship. A.A. acknowledges SABIC for the Career Development SABIC Chair. We 9 acknowledge Dr. Detlef-M. Smilgies for help with acquisition of GIWAXS data at CHESS. 10 CHESS is supported by the NSF \& NIH/NIGMS via NSF award DMR-1332208. We also 11 acknowledge Dr. Jianguo Mei for help with ZnPc purification and Dr. Lethy Krishnan 12 Jagadamma for helpful discussions.

13 


\section{References}

4

(1) Kan, B.; Zhang, Q.; Li, M.; Wan, X.; Ni, W.; Long, G.; Wang, Y.; Yang, X.; Feng, H.; Chen, Y. Solution-Processed Organic Solar Cells Based on Dialkylthiol-Substituted Benzodithiophene Unit with Efficiency near 10\%. J. Am. Chem. Soc., 2014, 136, 15529-15532.

(2) Press release (January 16, 2013) by Heliatek, http://www.heliatek.com; accessed: October 2014.

(3) Jagadamma, L. K.; Al-Senani, M.; El-Labban, A.; Gereige, I.; Ngongang Ndjawa, G. O.; Faria, J. C. D.; Kim, T.; Zhao, K.; Cruciani, F.; Anjum, D. H.; McLachlan, M. A.; Beaujuge, P. M.; Amassian, A. Polymer Solar Cells with Efficiency $>10 \%$ Enabled via a Facile Solution-Processed Al-Doped $\mathrm{ZnO}$ Electron Transporting Layer..Adv. Energy Mater. 2015, 5, 1500204.

(4) Liu, Y.; Zhao, J.; Li, Z.; Mu, C.; Ma, W.; Hu, H.; Jiang, K.; Lin, H.; Ade, H.; Yan, H.; Aggregation and morphology control enables multiple cases of highefficiency polymer solar cells. Nat. Commun., 2014, 5, 5293.

(5) Sullivan, P.; Jones, T. S.; Ferguson, A. J.; Heutz, S. Structural templating as a route to improved photovoltaic performance in copper phthalocyanine/fullerene (C60) heterojunctions. Appl. Phys. Lett. 2007, 91. 233114-1-233114-3.

(6) Rand, B. P.; Cheyns, D.; Vasseur, K.; Giebink, N. C.; Mothy, S.; Yi, Y.; Coropceanu, V.; Beljonne, D.; Cornil, J.; Brédas, J.-L.; Genoe, J. The Impact of Molecular Orientation on the Photovoltaic Properties of a Phthalocyanine/Fullerene Heterojunction. Adv. Funct. Mater. 2012, 22, $2987-$ 2995.

(7) Sakurai, T.; Fukasawa, R.; Saito, K.; Akimoto, K. Structural control of organic 
solar cells based on nonplanar metallophthalocyanine/C60 heterojunctions using organic buffer layers. Org. Electron. 2007, 8, 702-708.

(8) Vasseur, K.; Broch, K.; Ayzner, A. L.; Rand, B. P.; Cheyns, D.; Frank, C.; Schreiber, F.; Toney, M. F.; Froyen, L.; Heremans, P. Controlling the Texture and Crystallinity of Evaporated Lead Phthalocyanine Thin Films for NearInfrared Sensitive Solar Cells. Appl. Mater. Interfaces 2013, 5, 8505-8515.

(9) Yook, K. S.; Chin, B. D.; Lee, J. Y.; Lassiter, B. E.; Forrest, S. R. Vertical orientation of copper phthalocyanine in organic solar cells using a small molecular weight organic templating layer. Appl. Phys. Lett. 2011, 99, 043308.

(10) Cheng, C. H.; Wang, J.; Du, G. T.; Shi, S. H.; Du, Z. J.; Fan, Z. Q.;Bian, J. M.; Wang, M. S. Organic solar cells with remarkable enhanced efficiency by using a CuI buffer to control the molecular orientation and modify the anode. Appl. Phys. Lett. 2010, 97, 083305.

(11) Tumbleston, J. R.; Collins, B. A.; Yang, L.; Stuart, A. C.; Gann, E.; Ma, W.; You, W.; Ade, H. The influence of molecular orientation on organic bulk heterojunction solar cells. Nat. Photonics 2014, 8, 385.

(12) Rim, S. B.; Fink, R. F.; Schoneboom, J. C.; Erk, P.; Peumans, P. Effect of molecular packing on the exciton diffusion length in organic solar cells. Appl. Phys. Lett. 2007, 91, 173504.

(13) Matsushima, T.; Matsuo, H.; Yamamoto, T.; Nakao, A.; Murata, H. Horizontally oriented molecular thin films for application in organic solar cells. Solar Energy Materials \& Solar Cells 2014, 123, 81-91.

(14) Correia, H. M. G.; Barbosa, H. M. C.; Marques, L.; Ramos, M. M. D. Unravelling the effect of strand orientation on exciton migration in conjugated 25 
polymers. Comput. Mater. Sci. 2013, 75, 18-23.

(15) Lunt, R. R.; Benziger, J. B.; Forrest, S. R. Relationship between Crystalline Order and Exciton Diffusion Length in Molecular Organic Semiconductors. Adv. Mater. 2010, 22, 1233-1236.

(16) Chen, W.; Qi, D.-C.; Huang, H.; Gao, X.; Wee, A. T. S. Organic-Organic Heterojunction Interfaces: Effect of Molecular Orientation. Adv. Funct. Mater. 2011, 21, 410-424.

(17) Fu, Y. T.; Risko, C.; Brédas, J.-L. Intermixing at the Pentacene-Fullerene Bilayer Interface: A Molecular Dynamics Study. Adv. Mater. 2013, 25, 878882.

(18) Muccioli, L.; D'Avino, G.; Zannoni, C. Simulation of Vapor-Phase Deposition and Growth of a Pentacene Thin Film on C60 (001). Adv. Mater. 2011, 23, $4532-4536$.

(19) Bartelt, J. A.; Beiley, Z. M.; Hoke, E. T.; Mateker, W. R.; Douglas, J. D.; Collins, B. A.; Tumbleston, J. R.; Graham, K. R.; Amassian, A.; Ade, H.; Fréchet, J. M. J.; Toney, M. F.; McGehee, M. D. The Importance of Fullerene Percolation in the Mixed Regions of Polymer-Fullerene Bulk Heterojunction Solar Cells. Adv. Energy Mater. 2013, 3, 364-374.

(20) Westacott, P.; Tumbleston, J. R.; Shoaee, S.; Fearn, S.; Bannock, J. H.; Gilchrist, J. B.; Heutz, S.; deMello, J.; Heeney, M.; Ade H.; Durrant, J.; McPhail, D. S.; Stingelin, N. On the role of intermixed phases in organic photovoltaic blends. Energy Environ. Sci. 2013, 6, 2756-2764.

(21) Groves, C. Suppression of geminate charge recombination in organic photovoltaic devices with a cascaded energy heterojunction. Energy Environ. 
Sci. 2013, 6, 1546-1551.

(22) Schunemann, C.; Wynands, D.; Eichhorn, K. J.; Stamm, M.; Leo, K.; Riede, M. Evaluation and Control of the Orientation of Small Molecules for Strongly Absorbing Organic Thin Films. J. Phys. Chem. 2013, 117, 11600-11609.

(23) Zhou, Y.; Taima, T.; Miyadera, T.; Yamanari, T.; Kitamura, M.; Nakatsu, K.; Yoshida, Y. Glancing Angle Deposition of Copper Iodide Nanocrystals for Efficient Organic Photovoltaics. Nano Lett. 2012, 12, 4146-4152.

(24) Copper, A.; Laibinis, P. E.; Bain, C. D.; Whitesides, M. Attenuation of photoelectrons in monolayers of n-alkanethiols adsorbed on copper, silver, and gold. Phys Chem, 1991, 95, 7017-7021.

(25) Monjushiro, H.; Watanabe, I. Probing Depth of Ultraviolet Photoelectron Yield Spectroscopy in Hydrocarbon Films. Anal. Sci. 1995, 11, 797-800.

(26) Sushko, G. B.; Verkhovtsev, A.V.; Yakubovich, A.V.; Schramm, S.; Solov'yov, A.V. Molecular Dynamics Simulation of Self-Diffusion Processes in Titanium in Bulk Material, on Grain Junctions and on Surface. J. Phys. Chem. A 2014, $118,6685-6691$.

(27) Zhu, L.; Brian, C. W.; Swallen, S. F.; Straus, P. T.; Ediger, M. D. Surface SelfDiffusion of an Organic Glass. Phys. Rev. Lett, 2011, 106, 256103.

(28) Capaccioli, S.; Ngai, K. L.; Paluch, M.; Prevosto, D. Mechanism of fast surface self-diffusion of an organic glass. Phys. Rev. E 2012, 86, 051503.

(29) Vandewal, K.; Tvingstedt, K.; Gadisa, A.; Inganäs, O.; Manca, J. V. Relating the open-circuit voltage to interface molecular properties of donor:acceptor bulk heterojunction solar cells. Phys. Rev. B 2010, 81, 125204.

(30) Zhao, K.; Khan, H. U.; Li R.; Su, Y.; Amassian, A. Entanglement of 27 ACS Paragon Plus Environment 
1 Conjugated Polymer Chains Influences Molecular Self-Assembly and Carrier

2 Transport. Adv. Funct. Mater. 2013, 23, 6024-6035.

(31) Hoofman, R. J. O. M.; de Haas, M. P.; Siebbeles, L. D. A.; Warman, J. M. Highly mobile electrons and holes on isolated chains of the semiconducting polymer poly(phenylene vinylene). Nature 1998, 392, 54-56.

(32) Tseng, H. R.; Phan, H.; Luo, C.; Wang, M.; Perez, L. A.; Patel, S. N.; Ying, L.; Kramer, E. J.; Nguyen, T. Q.; Bazan, G. C.; Heeger, A. J. High-Mobility FieldEffect Transistors Fabricated with Macroscopic Aligned Semiconducting Polymers. Adv. Mater. 2014, 26, 2993-2998.

(33) Burke, T. M.; McGehee, M. D. How High Local Charge Carrier Mobility and an Energy Cascade in a Three-Phase Bulk Heterojunction Enable $>90 \%$ Quantum Efficiency. Adv. Mater. 2014, 26, 1923-1928.

(34) Kim, H. J.; Kim, J. W.; Lee, H. H.; Lee, B.; Kim, J.-J. Initial Growth Mode, Nanostructure, and Molecular Stacking of a ZnPc:C60 Bulk Heterojunction. Adv. Funct. Mater. 2012, 22, 4244-4248.

(35) Hedley, G. J.; Ward, A. J.; Alekseev, A.; Howells, C. T.; Martins, E. R.; Serrano, L. A.; Cooke, G.; Ruseckas, A.; Samuel, I. D. W.; Determining the optimum morphology in high-performance polymer-fullerene organic photovoltaic cells. Nat. Commun. 2013, 4, 2867.

(36) Bartesaghi, D.; Koster, L. J. A. The Effect of Large Compositional Inhomogeneities on the Performance of Organic Solar Cells: A Numerical Study. Adv. Funct. Mater. 2014, 25, 2013-2013

(37) Xiao, T.; Xu, H.; Grancini, G.; Mai, J.; Petrozza, A.; Jeng, U.-S.; Wang, Y.; Xin, X.; Lu, Y.; Choon, N. S.; Xiao, H.; Ong, B. S.; Lu X.; Zhao, N. Molecular 28 ACS Paragon Plus Environment 
1

2

3

4

5

6

7

8

9

10

11

12

13

14

15

16

17

18

19

20

21

22

23

24

25

26

27

28

29

30

31

32

33

34

35

36

37

38

39

40

41

42

43

44

45

46

47

48

49

50

51

52

53

54

55

56

57

58

59

60

1 Packing and Electronic Processes in Amorphous-like Polymer Bulk

2 Heterojunction Solar Cells with Fullerene Intercalation. Sci. Rep., 2014, 4,

3 5211.

4

29

ACS Paragon Plus Environment

ACS Paragon Plus Environment 
1 Table of Contents Graphic (ToC Image)

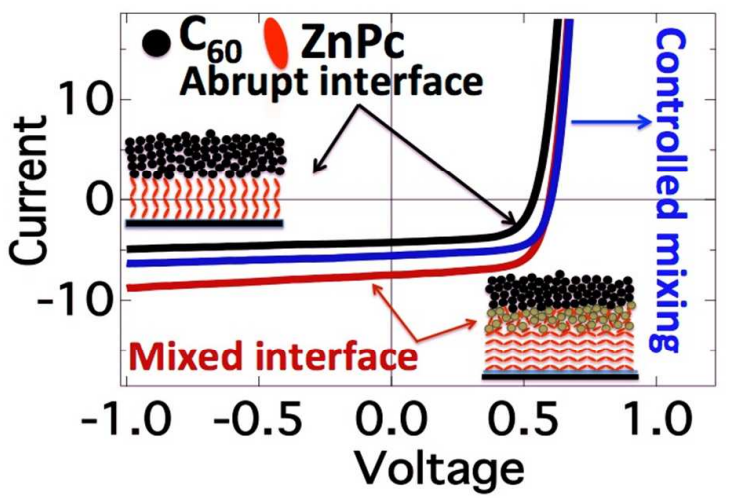

2

3

4 